\title{
AMCoR
}

Asahikawa Medical University Repository http://amcor.asahikawa-med.ac.jp/

Neuroscience Letters (2012.10) 527(1):28-33.

Nerve injury induces the expression of syndecan-1 heparan sulfate proteoglycan in peripheral motor neurons

Koichi Murakami, Shigetaka Yoshida 
Original Research Article

\section{Nerve injury induces the expression of syndecan-1 heparan sulfate proteoglycan in peripheral motor neurons}

Koichi Murakami, Shigetaka Yoshida

Department of Functional Anatomy and Neuroscience, Asahikawa Medical University

\section{Corresponding author:}

Koichi Murakami, M.D., Ph.D.

Department of Functional Anatomy and Neuroscience, Asahikawa Medical University

2-1-1-1 Midorigaoka-Higashi, Asahikawa 078-8510, Japan

Tel: +81-166-68-2303; Fax: +81-166-68-2309

E-mail: kmura@asahikawa-med.ac.jp 


\section{Abstract}

Heparan sulfate proteoglycans play important roles in embryogenesis, including the development of the central nervous system. However, their function in nerve regeneration is not yet understood. We previously reported that nerve injury induces the expression of heparan sulfate glycosaminoglycans and syndecan-1, a heparan sulfate proteoglycan, in injured hypoglossal motor neurons. In this study, we examined the expression of syndecan family members, including syndecan-1, in injured hypoglossal motor neurons after hypoglossal nerve axotomy. We could not detect any changes in expression after axotomy, except for syndecan-1. The expression of syndecan-1 was markedly increased on post-operative day 7. Syndecan-1 was localized not only in the cell bodies of hypoglossal motor neurons, but also in the injured hypoglossal nerve, and it accumulated in the terminals of regenerating fibers. Similarly, facial nerve axotomy and vagus nerve axotomy induced the expression of syndecan-1 in the facial nucleus, dorsal nucleus of vagus and ambiguous nucleus, respectively. However, sciatic nerve axotomy induced very little syndecan-1 expression in injured spinal motor neurons. These results suggest that syndecan-1 may have a crucial role in the survival of injured motor neurons and in nerve regeneration after injury. Our observations also reveal the diversity of peripheral motor neurons. 


\section{Keywords:}

syndecan; heparan sulfate proteoglycan; nerve injury; nerve regeneration

\section{Abbreviations:}

ECM, extracellular matrix; HSPG, heparan sulfate proteoglycan; HS-GAG, heparan sulfate glycosaminoglycan; CNS, central nervous system; ATF3, activating transcription factor 3; GFAP, glial fibrillary acidic protein; PB, phosphate buffer; RT-PCR, reverse transcription-polymerase chain reaction; GAPDH, glyceraldehyde-3-phosphate dehydrogenase; PBS, phosphate-buffered saline; DAB, 3,3'-diaminobenzidine; POD, post operative day; FGF2, fibroblast growth factor 2; nNOS, neuronal nitric oxide synthase. 


\section{Introduction}

Axonal regeneration after nerve injury is a very complex process, which involves the detection of axonal injury, changes in gene expression, inflammatory responses, cytoskeletal changes, as well as interactions with glial cells, inflammatory cells and extracellular matrix (ECM) [22]. ECM components play critical roles, and either promote or inhibit axonal regeneration $[4,10,11]$. Heparan sulfate proteoglycans (HSPGs) are glycoproteins containing heparan sulfate glycosaminoglycans (HS-GAGs), which are long sulfated sugar chains. HS-GAGs consist of repeating disaccharide units composed of D-glucosamine and D-glucuronic acid or L-iduronic acid [1]. HSPGs have a variety of functions in numerous tissues, affecting cell growth, cell adhesion, cell migration, angiogenesis and coagulation [1, 9, 20]. In the nervous system, HSPGs are involved in several processes, including the patterning of the central nervous system (CNS) [14], axon guidance [19, 26], synaptogenesis [16] and synaptic plasticity [17, 24$]$.

Syndecans are a major family of HSPGs comprised of four members in mammals. They are type- 1 transmembrane proteins and are composed of three domains; an extracellular domain, which is variable for each syndecan, a transmembrane domain, which is conserved among syndecans, and a short cytoplasmic domain. HS-GAGs are attached to the extracellular domains where they mediate their functions [5]. 
Syndecan-1/CD138 was originally isolated from normal murine mammary gland (NMuMG) cells [25]. In physiological conditions, syndecan-1 is mainly expressed in epithelial tissues, such as epidermis and cornea, and plasma cells [2]. Syndecan-1 is also expressed in the embryonic CNS, particularly strongly in neuroepithelial cells [8]. Moreover, syndecan-1 is expressed in several pathological conditions, such as breast and prostate cancers [6], skin wounds [1] and following brain injury [15].

We previously reported that hypoglossal nerve injury induces the expression of EXT2, a glycosyltransferase required for HS-GAG synthesis, in the injured hypoglossal motor neurons, and the amount of HS-GAG is increased in the hypoglossal nucleus ipsilateral to the lesion [21]. In the same report, we also showed that syndecan-1 mRNA was expressed in the injured hypoglossal motor neurons, but not in the intact motor neurons, suggesting the possibility that syndecan-1 and its HS-GAG play functional roles in the regeneration of injured axons.

In this study, we investigated in detail the expression and distribution of syndecan- 1 in the hypoglossal nucleus and hypoglossal nerve after hypoglossal nerve axotomy. Moreover, we investigated the expression of syndecan-1 in the facial nucleus, dorsal nucleus of vagus, ambiguous nucleus and spinal motor neurons after facial nerve axotomy, vagus nerve axotomy and sciatic nerve axotomy, respectively.

\section{Materials and methods}


In all experiments, we used 6 to 8-week-old BALB/c mice. Before axotomy, mice were anesthetized with sodium pentobarbital $(50 \mathrm{mg} / \mathrm{kg})$. Nerves were cut with a pair of scissors. For hypoglossal nerve axotomy, an incision was made in the skin of the neck, and the right hypoglossal nerve was cut. For facial nerve axotomy, an incision was made in the skin of the left temporal region, and a branch of the left facial nerve was cut in front of the parotid gland. For vagus nerve axotomy, an incision was made in the skin of the neck, and the right vagus nerve along with the common carotid artery was cut. For sciatic nerve axotomy, an incision was made in the skin of the right mid-thigh, and the right sciatic nerve was cut. All experiments were conducted in accordance with the guidelines published in the U.S. National Institutes of Health Guide for the Care and Use of Laboratory Animals, implemented by Asahikawa Medical University. All efforts were made to minimize the number of animals used and their pain and suffering.

For in situ hybridization, the following cDNA fragments were used as probes: syndecan-1 (GenBank ID: NM_011519): nucleotides 358-677; syndecan-2 (GenBank ID: NM_008304): nucleotides 693-1017; syndecan-3 (GenBank ID: NM_011520): nucleotides 292-593; syndecan-4 (GenBank ID: NM_011521): nucleotides 131-425; activating transcription factor 3 (ATF3) (GenBank ID: AY329367): nucleotides 23-460. In situ hybridization was performed using digoxigenin (Roche Diagnostics, Indianapolis, IN, USA)-labeled cRNA probes. Hybridization was performed on fresh-frozen mouse brain 
sections (18- $\mu$ m-thick) at $0,1,3,7,14$ and $28 \mathrm{~d}$ after hypoglossal nerve axotomy, using a previously described method [30].

For mRNA detection in the axotomized hypoglossal nuclei, reverse transcription-polymerase chain reaction (RT-PCR) was performed on 4 mice for each time point examined. The mice were killed by decapitation on $0,1,3,7,14$ and $28 \mathrm{~d}$ after axotomy, and the hypoglossal nuclei were harvested. Total RNA was extracted from the samples using TRIzol reagent (Life Technologies, Carlsbad, CA, USA). Aliquots containing $2 \mu \mathrm{g}$ of total RNA were used for RT with AMV Reverse Transcriptase (Promega, Madison, USA) according to the manufacturer's protocol. Aliquots from the RT reaction were then amplified by PCR. The primers used and their product sizes were as follows: syndecan-1: forward, 5'-CATGAGACCTCGGCTCCCAC-3', reverse, 5'-TTCCTTCCTGTCCAAAAGGC-3' (270 bp); ATF3: forward, 5'-GATGCTTCAACATCCAGGCCAGG-3', reverse, 5'-TTAGCTCTGCAATGTTCCTTC-3' (438 bp); glyceraldehyde-3-phosphate dehydrogenase (GAPDH): forward, 5'-CTACATGGTCTACCTGTTCCAGT-3', reverse, 5'-AGTTGTCATGGATGACCTTGG-3' (380 bp). The number of PCR cycles used for the amplifications were: 35 cycles for syndecan-1; 32 cycles for ATF-3; 25 cycles for GAPDH. The reaction conditions were as follows: denaturation at $94{ }^{\circ} \mathrm{C}$ for $30 \mathrm{~s}$, annealing at $62{ }^{\circ} \mathrm{C}$ (syndecan-1) or $60{ }^{\circ} \mathrm{C}$ (ATF3 and GAPDH) for $30 \mathrm{~s}$, and extension at $72{ }^{\circ} \mathrm{C}$ for $30 \mathrm{~s}$. The reaction products were separated by electrophoresis in 1.5\% agarose gels, and visualized 
using a transilluminator after staining with ethidium bromide. For quantitative analysis, the LightCycler rapid thermal system (Roche Diagnostics) was used according to the manufacturer's protocol. The primers used and PCR conditions were the same as above. A serially-diluted cDNA from mouse kidney was used as a standard. The quantified data were analyzed with the LightCycler analysis software. Differences in expression during the various time points were assessed using ANOVA with post hoc Tukey-Kramer test.

For immunohistochemistry, mice were fixed with $4 \%$ paraformaldehyde in $0.1 \mathrm{M}$ phosphate buffer ( $\mathrm{PB}, \mathrm{pH} 7.4$ ) by transcardial perfusion at $0,1,3,7,14$ and $28 \mathrm{~d}$ after axotomy. The tissues were removed, postfixed overnight in the same fixative and immersed in $20 \%$ sucrose in $0.1 \mathrm{M}$ PB for 2-3 days. Next, the samples were frozen in powdered dry ice and sectioned using a cryostat. The sections (18- $\mu$ m-thick) were treated with $0.3 \% \mathrm{H}_{2} \mathrm{O}_{2}$ for 5 min, washed with $0.1 \mathrm{M}$ phosphate-buffered saline (PBS; pH 7.4), blocked with 5\% bovine serum albumin in 0.1 M PBS for $1 \mathrm{~h}$ and incubated with an anti-syndecan-1 antibody (281-2, rat monoclonal, BD Bioscience, Franklin Lakes, New Jersey, USA; 1:2,000), an anti-ATF3 antibody (rabbit polyclonal, sc-188, Santa Cruz Biotechnology, Santa Cruz, California, USA; 1:500), an anti-glial fibrillary acidic protein (GFAP) antibody (rabbit polyclonal, G9269, Sigma, Saint Louis, Missouri, USA; 1:500), an anti-Iba1 antibody (rabbit polyclonal, 019-19741, Wako, Osaka, Japan; 1:500) and an anti-tubulin beta-3 chain antibody (TUJ1, rabbit monoclonal, MRB-435P, Covance, new Jersey, USA; $1: 500$ ) at $4{ }^{\circ} \mathrm{C}$ overnight. After a 
wash with 0.1 M PBS, the sections were incubated with a biotinylated IgG (Vector Laboratories, Burlingame, CA, USA; 1:500) for 3,3'-diaminobenzidine (DAB) visualization or Alexa 488 or 568-conjugated secondary antibodies (Life Technologies, 1:1,000) for $2 \mathrm{~h}$ at room temperature. For DAB visualization, the sections were incubated with an $A B C$ reagent mixture (Vector Laboratories) for $2 \mathrm{~h}$ at $4{ }^{\circ} \mathrm{C}$ and visualized using DAB (DOJINDO LABORATORIES, Kumamoto, Japan). To evaluate the proportion of syndecan-1-positive motor neurons, we used three mice in each experiments.

\section{Results}

Because we previously reported that syndecan- 1 mRNA is induced in hypoglossal motor neurons after hypoglossal nerve axotomy [21], we first investigated the mRNA expression of other syndecan family members in the hypoglossal nuclei 7 days after hypoglossal nerve axotomy using in situ hybridization. Syndecan-1 mRNA was strongly expressed in the hypoglossal nucleus ipsilateral to the lesion, but not in the contralateral nucleus (Fig. 1A). However, we were unable to detect mRNA for syndecan-2, 3 or 4 in either the ipsilateral or contralateral hypoglossal nucleus (Fig. 1B-D).

To evaluate changes in syndecan-1 mRNA expression after axotomy, we examined the amount of mRNA in the hypoglossal nucleus ipsilateral to the lesion using RT-PCR (Fig.1E, F). Prior to axotomy, syndecan-1 mRNA was not detected in the hypoglossal nucleus. 
Syndecan-1 mRNA expression was induced in the hypoglossal nucleus on post-operative day (POD) 1 after axotomy. It significantly increased by POD 7, and then gradually decreased until the last day examined, POD 28. RNA from skin was used as a positive control for the syndecan-1 RT-PCR. ATF3 was used as a marker of injured neurons.

Next, we performed in situ hybridization to identify cells expressing syndecan-1 mRNA (Fig. 1G-N). Syndecan-1 mRNA was not detected in the hypoglossal nucleus prior to axotomy, consistent with the RT-PCR result (Fig. 1G). On PODs 3 and 7, the number and intensity of the hybridization signals were markedly increased in the hypoglossal nucleus ipsilateral to the lesion. Subsequently, the hybridization signal decreased until POD 28 (Fig. 1I-L). We could not detect any hybridization signal in the contralateral nucleus at any time point examined (Fig. 1M). The hybridization signals were observed in large cells, corresponding to typical motor neurons in the nucleus, but not in surrounding glial cells.

We also examined the expression of syndecan-1 protein by immunohistochemistry using a syndecan-1-specific antibody (Fig. 2). Consistent with the RT-PCR and in situ hybridization results, syndecan-1 protein was not detected in the hypoglossal nucleus prior to axotomy (Fig. 2A). The expression of syndecan-1 protein was observed in the hypoglossal nucleus ipsilateral to the lesion on POD 1 (Fig. 2B), and was markedly increased on PODs 7 and 14 (Fig. 2D, E). On POD 28, we could not detect any signal for syndecan-1 protein in the ipsilateral nucleus (Fig. 2F). There was no signal in the contralateral nucleus at any time 
point examined (Fig. 2G). The syndecan-1-expressing cells were large motor neurons, as observed with in situ hybridization (Fig. 1I-L). The GFAP immunoreactivity, a marker of astrocyte, was not colocalized with syndecan-1. A part of Iba1 immunoreactivity, a marker of microglia, was seemingly colocalized with syndecan- 1 around the motor neurons, possibly due to close apposition of microglial processes to damaged motor neurons (Supplementary Figure 1).

After hypoglossal nerve axotomy, we could detect syndecan-1 protein labeling along the hypoglossal nerve tract in the medulla (data not shown). This indicates transport of syndecan-1 along injured nerves; therefore, we investigated the distribution of syndecan-1 protein in these nerves using immunohistochemistry (Fig. 2H-K). In the intact hypoglossal nerve, the epineurium and vascular structures were stained; however, no syndecan-1-positive nerve fibers were detected (Fig. 2H). On POD 7, many syndecan-1-positive fibers were observed proximal to the lesion (Fig. 2I). The number of syndecan-1-positive fibers and the intensity of syndecan-1 immunoreactivity in the distal stump of the injured nerve were greater than in the more proximal portion (Fig. 2J). We could also detect several fibers exhibiting strong syndecan-1 immunoreactivity in the distal portion (Fig. 2K). The syndecan-1 immunoreactivity was colocalized with tubulin beta-3 chain immunoreactivity, a marker of neurons (Supplementary Figure 2). 
To determine whether syndecan-1 expression after nerve injury is specific to hypoglossal motor neurons, we investigated the expression of the proteoglycan in the facial nucleus, dorsal nucleus of vagus, ambiguous nucleus and spinal motor neurons after facial nerve axotomy, vagus nerve axotomy and sciatic nerve axotomy, respectively. Prior to axotomy, none of the motor neurons examined expressed syndecan-1 protein (Fig. 3A, E, I, M, Q). On POD 7, hypoglossal nerve axotomy, facial nerve axotomy and vagus nerve axotomy induced the expression of ATF3 protein in the hypoglossal nucleus, facial nucleus, dorsal nucleus of vagus and ambiguous nucleus ipsilateral to the lesion, respectively (Fig. 3C, G, K, O), and syndecan-1 protein was expressed in ATF3-positive motor neurons (Fig. 3B-D, F-H, J-L, N-P). Interestingly, not all ATF3-positive motor neurons expressed syndecan-1 protein (Fig. 3D, H, L, arrow). The proportion of syndecan-1-positive neurons to ATF3-positive neurons was $55.1 \pm 9.6 \%$ (mean \pm standard deviation) in the hypoglossal nucleus, $36.7 \pm 5.6 \%$ in the facial nucleus, $72.9 \pm 4.9 \%$ in the dorsal nucleus of vagus and $71.8 \pm 7.5 \%$ in the ambiguous nucleus. Although sciatic nerve axotomy also induced the expression of ATF3 protein in spinal motor neurons ipsilateral to the lesion (Fig. 3S), only a few ATF3-positive motor neurons expressed syndecan-1 protein on POD 7 (3.1 $\pm 2.0 \%$, Fig. 3R-T), and the intensity of syndecan-1 immunoreactivity was very weak. A very low level of syndecan-1 expression in spinal motor neurons was observed not only on POD 7, but also on PODs 1, 3, 14 and 28 (data not shown). We also examined the expression of syndecan-1 mRNA in injured spinal 
motor neurons by in situ hybridization. We could detect few hybridization signals of syndecan-1 mRNA in spinal motor neurons ipsilateral to the lesion compared with injured hypoglossal motor neurons (Supplementary Figure 3).

\section{Discussion}

In this study, we examined the expression profile of syndecan-1 in injured hypoglossal motor neurons. Syndecan-1 expression was induced in injured hypoglossal motor neurons after axotomy and was most intense on POD 7. Syndecan-1 mRNA and protein levels declined until the last day examined, POD 28, when the expression was comparable to that in intact motor neurons (Figs. 1 and 2). On POD 28, we could detect several fibers re-innervating the tongue muscle [18] (our unpublished data). This suggests that injury-induced syndecan-1 may play a role in the survival of injured motor neurons and in the regeneration of the injured nerve.

Syndecan-1 functions as a co-receptor for fibroblast growth factor 2 (FGF2) [27]. FGF2 mRNA and protein are highly expressed in injured hypoglossal motor neurons [13], providing trophic support to these cells $[28,29]$. Collectively, these previous observations and our present results suggest that injury-induced syndecan-1 may act as a co-receptor for FGF2 in injured hypoglossal motor neurons, thereby enhancing the ability of FGF2 to promote the survival of these cells. 
We could detect syndecan-1 protein not only in the hypoglossal nucleus, but also in the injured hypoglossal nerve (Fig. 2I-K). The number of syndecan-1-positive fibers and the intensity of syndecan-1 immunoreactivity in the distal stump of the injured nerve were greater than in the more proximal portion (Fig. 2I, J). Moreover, several fibers in the distal portion displayed strong syndecan-1 immunoreactivity (Fig. 2K), suggesting that syndecan-1 expressed in injured motor neurons is transported down the hypoglossal nerve and accumulates in the terminals of regenerating fibers. Since nerve injury induces the expression of FGF2 mRNA in the lesion [12, 13], expressed syndecan-1 may act as a co-receptor for FGF2, not only in the hypoglossal nucleus, but also in the nerve lesion, and promote the survival of injured motor neurons. Application of HS-GAG to the site of the lesion enhances myelination of injured axons and helps restore conduction velocity in the injured sciatic nerve [3]. Our present results suggest that syndecan-1, transported to the lesion, may also promote nerve regeneration, in addition to enhancing neuronal survival. Furthermore, it has been reported that heparan sulfate proteoglycans, including Drosophila syndecan, are important for axon guidance during development of the nervous system [19, 23, 26]. Thus, syndecan-1, accumulated in the terminals of regenerating fibers, may function in axon guidance to promote proper innervation.

Facial nerve axotomy and vagus nerve axotomy also induced the expression of syndecan-1 in injured motor neurons; however, it only very weakly induced expression of the 
proteoglycan in injured spinal motor neurons (Fig. 3, Supplementary Figure 3). A similar phenomenon has been reported for the expression of neuronal nitric oxide synthase (nNOS) after nerve injury in rats. Facial, vagus and hypoglossal nerve axotomy induced the expression of nNOS in injured motor neurons [32]. However, injury to the sciatic nerve or ventral root did not induce nNOS expression in injured spinal motor neurons [7, 31]. These results suggest that cranial motor neurons and spinal motor neurons react differently to nerve injury. Moreover, not all injured motor neurons expressed syndecan-1, even in the hypoglossal nucleus, facial nucleus or dorsal nucleus of vagus (Fig. 3D, H, L, arrows). This is indicative of the diversity of motor neurons within the cranial nervous system.

\section{Conclusions}

In this study, we suggest that syndecan-1 may have functional roles in the survival of injured motor neurons and in nerve regeneration in the peripheral nervous system. Syndecan-1 may provide a novel target for therapeutic strategies for nerve and CNS injuries.

\section{Acknowledgments}

We are grateful to Mr. T. Sasaki and Mr. K. Hazawa for technical assistance. This study was supported by Asahikawa Medical University. The authors declare no financial interests. 


\section{References}

[1] M. Bernfield, M. Gotte, P.W. Park, O. Reizes, M.L. Fitzgerald, J. Lincecum, M. Zako, Functions of cell surface heparan sulfate proteoglycans, Annu Rev Biochem 68 (1999) 729-777.

[2] M. Bernfield, R. Kokenyesi, M. Kato, M.T. Hinkes, J. Spring, R.L. Gallo, E.J. Lose, Biology of the syndecans: a family of transmembrane heparan sulfate proteoglycans, Annu Rev Cell Biol 8 (1992) 365-393.

[3] C.H. Chau, D.K. Shum, Y.S. Chan, K.F. So, Heparan sulphates upregulate regeneration of transected sciatic nerves of adult guinea-pigs, Eur J Neurosci 11 (1999) 1914-1926.

[4] Z.L. Chen, W.M. Yu, S. Strickland, Peripheral regeneration, Annu Rev Neurosci 30 (2007) 209-233.

[5] J.R. Couchman, Syndecans: proteoglycan regulators of cell-surface microdomains?, Nat Rev Mol Cell Biol 4 (2003) 926-937.

[6] C.Y. Fears, A. Woods, The role of syndecans in disease and wound healing, Matrix Biol 25 (2006) 443-456.

[7] C.E. Fiallos-Estrada, W. Kummer, B. Mayer, R. Bravo, M. Zimmermann, T. Herdegen, Long-lasting increase of nitric oxide synthase immunoreactivity, 
NADPH-diaphorase reaction and c-JUN co-expression in rat dorsal root ganglion neurons following sciatic nerve transection, Neuroscience letters 150 (1993) 169-173.

[8] M. Ford-Perriss, K. Turner, S. Guimond, A. Apedaile, H.D. Haubeck, J. Turnbull, M. Murphy, Localisation of specific heparan sulfate proteoglycans during the proliferative phase of brain development, Dev Dyn 227 (2003) 170-184.

[9] M.M. Fuster, L. Wang, Endothelial heparan sulfate in angiogenesis, Prog Mol Biol Transl Sci 93 (2010) 179-212.

[10] C.M. Galtrey, J.W. Fawcett, The role of chondroitin sulfate proteoglycans in regeneration and plasticity in the central nervous system, Brain Res Rev 54 (2007) 1-18.

[11] B. Grimpe, J. Silver, The extracellular matrix in axon regeneration, Progress in brain research 137 (2002) 333-349.

[12] C. Grothe, C. Meisinger, A. Hertenstein, H. Kurz, K. Wewetzer, Expression of fibroblast growth factor-2 and fibroblast growth factor receptor 1 messenger RNAs in spinal ganglia and sciatic nerve: regulation after peripheral nerve lesion, Neuroscience 76 (1997) 123-135.

[13] K. Huber, C. Meisinger, C. Grothe, Expression of fibroblast growth factor-2 in hypoglossal motoneurons is stimulated by peripheral nerve injury, J Comp Neurol 382 (1997) 189-198. 
[14] M. Inatani, F. Irie, A.S. Plump, M. Tessier-Lavigne, Y. Yamaguchi, Mammalian brain morphogenesis and midline axon guidance require heparan sulfate, Science 302 (2003) 1044-1046.

[15] K. Iseki, S. Hagino, T. Mori, Y. Zhang, S. Yokoya, H. Takaki, C. Tase, M. Murakawa, A. Wanaka, Increased syndecan expression by pleiotrophin and FGF receptor-expressing astrocytes in injured brain tissue, Glia 39 (2002) 1-9.

[16] G.J. Jenniskens, J.H. Veerkamp, T.H. van Kuppevelt, Heparan sulfates in skeletal muscle development and physiology, J Cell Physiol 206 (2006) 283-294.

[17] M. Kaksonen, I. Pavlov, V. Voikar, S.E. Lauri, A. Hienola, R. Riekki, M. Lakso, T. Taira, H. Rauvala, Syndecan-3-deficient mice exhibit enhanced LTP and impaired hippocampus-dependent memory, Mol Cell Neurosci 21 (2002) 158-172.

[18] D. Kittaka, M. Itoh, Y. Ohmi, Y. Kondo, S. Fukumoto, T. Urano, O. Tajima, K. Furukawa, Impaired hypoglossal nerve regeneration in mutant mice lacking complex gangliosides: down-regulation of neurotrophic factors and receptors as possible mechanisms, Glycobiology 18 (2008) 509-516.

[19] J.S. Lee, S. von der Hardt, M.A. Rusch, S.E. Stringer, H.L. Stickney, W.S. Talbot, R. Geisler, C. Nusslein-Volhard, S.B. Selleck, C.B. Chien, H. Roehl, Axon sorting in the optic tract requires HSPG synthesis by ext2 (dackel) and extl3 (boxer), Neuron 44 (2004) 947-960. 
[20] J. Liu, L.C. Pedersen, Anticoagulant heparan sulfate: structural specificity and biosynthesis, Appl Microbiol Biotechnol 74 (2007) 263-272.

[21] K. Murakami, K. Namikawa, T. Shimizu, T. Shirasawa, S. Yoshida, H. Kiyama, Nerve injury induces the expression of EXT2, a glycosyltransferase required for heparan sulfate synthesis, Neuroscience 141 (2006) 1961-1969.

[22] G. Raivich, M. Makwana, The making of successful axonal regeneration: genes, molecules and signal transduction pathways, Brain Res Rev 53 (2007) 287-311.

[23] J.M. Rawson, B. Dimitroff, K.G. Johnson, X. Ge, D. Van Vactor, S.B. Selleck, The heparan sulfate proteoglycans Dally-like and Syndecan have distinct functions in axon guidance and visual-system assembly in Drosophila, Curr Biol 15 (2005) 833-838. O. Reizes, S.C. Benoit, D.J. Clegg, The role of syndecans in the regulation of body weight and synaptic plasticity, Int J Biochem Cell Biol 40 (2008) 28-45.

[25] S. Saunders, M. Jalkanen, S. O'Farrell, M. Bernfield, Molecular cloning of syndecan, an integral membrane proteoglycan, J Cell Biol 108 (1989) 1547-1556. P. Steigemann, A. Molitor, S. Fellert, H. Jackle, G. Vorbruggen, Heparan sulfate proteoglycan syndecan promotes axonal and myotube guidance by slit/robo signaling, Curr Biol 14 (2004) 225-230. 
[27] R. Steinfeld, H. Van Den Berghe, G. David, Stimulation of fibroblast growth factor receptor-1 occupancy and signaling by cell surface-associated syndecans and glypican, J Cell Biol 133 (1996) 405-416.

[28] Y.D. Teng, I. Mocchetti, A.M. Taveira-DaSilva, R.A. Gillis, J.R. Wrathall, Basic fibroblast growth factor increases long-term survival of spinal motor neurons and improves respiratory function after experimental spinal cord injury, J Neurosci 19 (1999) 7037-7047. [29] Y.D. Teng, I. Mocchetti, J.R. Wrathall, Basic and acidic fibroblast growth factors protect spinal motor neurones in vivo after experimental spinal cord injury, Eur J Neurosci 10 (1998) 798-802.

[30] R. Terayama, Y. Bando, T. Takahashi, S. Yoshida, Differential expression of neuropsin and protease $\mathrm{M} /$ neurosin in oligodendrocytes after injury to the spinal cord, Glia 48 (2004) 91-101.

[31] W. Wu, Expression of nitric-oxide synthase (NOS) in injured CNS neurons as shown by NADPH diaphorase histochemistry, Exp Neurol 120 (1993) 153-159.

[32] W.H. Yu, Nitric oxide synthase in motor neurons after axotomy, J Histochem Cytochem 42 (1994) 451-457. 


\section{Figure legends}

Fig. 1. Expression of syndecan mRNA in the hypoglossal nucleus. (A-D) Expression of syndecan-1 (A), syndecan-2 (B), syndecan-3 (C) and syndecan-4 (D) mRNA in the hypoglossal nucleus 7 days after axotomy, examined by in situ hybridization. (E, F) Representative images (E) and Quantification of the amount of syndecan-1 mRNA (F) evaluated by RT-PCR. Statistical analyses were performed between all groups $(* P<0.01$, ANOVA with post hoc Tukey-Kramer test). (G-M) Representative images of in situ hybridization for syndecan-1 in intact (G), ipsilateral (H, POD 1; I, POD 3; J, POD 7; K, POD 14; L, POD 28) and contralateral (M, POD 7) hypoglossal nuclei. (N) In situ hybridization images for ATF3 in the ipsilateral hypoglossal nucleus (POD 7). Scale bar = $200 \mu \mathrm{m}(\mathrm{A}-\mathrm{D})$ and $100 \mu \mathrm{m}(\mathrm{G}-\mathrm{N})$.

Fig. 2. Immunohistochemical analysis of syndecan-1 expression. (A-G) Representative images in intact (A), ipsilateral (B, POD 1; C, POD 3; D, POD 7; E, POD 14; F, POD 28) and contralateral (G, POD 7) hypoglossal nuclei. (H-K) Representative images of the intact hypoglossal nerve $(\mathrm{H})$, proximal region (I), distal stump (J) and distal region (K) of injured hypoglossal nerve on POD 7. Arrows indicate syndecan-1-positive fibers. Scale bar $=100 \mu \mathrm{m}$ (A-G), $200 \mu \mathrm{m}(\mathrm{H})$ and $50 \mu \mathrm{m}(\mathrm{I}-\mathrm{K})$. 
Fig. 3. Immunohistochemical analysis of syndecan-1 expression in the hypoglossal nucleus (A-D), facial nucleus (E-H), dorsal nucleus of vagus (I-L), ambiguous nucleus (M-P) and spinal motor neurons (Q-T). Panels A, E, I, M and Q show intact nuclei, and the other panels show the nuclei ipsilateral to the lesions 7 days after axotomy. Panel C, G, K, O and S show ATF3 immunohistochemistry, and the other panels show syndecan-1 immunohistochemistry. Arrows in panels D, H and L show ATF3 ${ }^{+} /$syndecan $-1^{-}$motor neurons. Scale bar $=50 \mu \mathrm{m}$. 


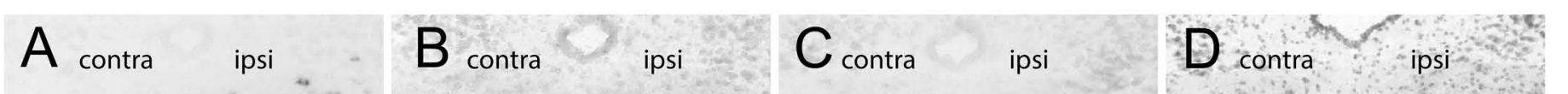

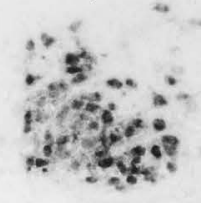

syndecan-1

E

$\begin{array}{llll} & \text { intact } 1 d \text { 3d } & \\ \text { Syndecan-1 } & & \\ \text { ATF-3 } & & \\ \text { GAPDH } & \end{array}$ syndecan-2

\section{syndecan-3}

syndecan-4

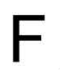

Skin

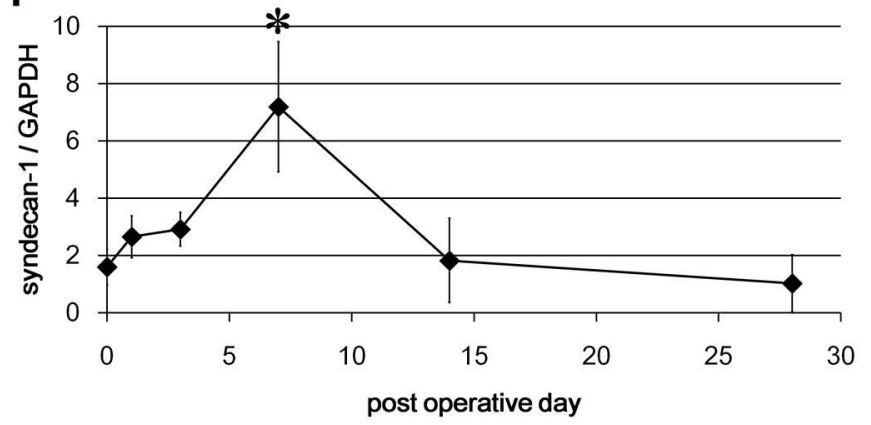

G

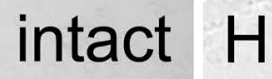

1d I

3d J

$7 d$

(9)
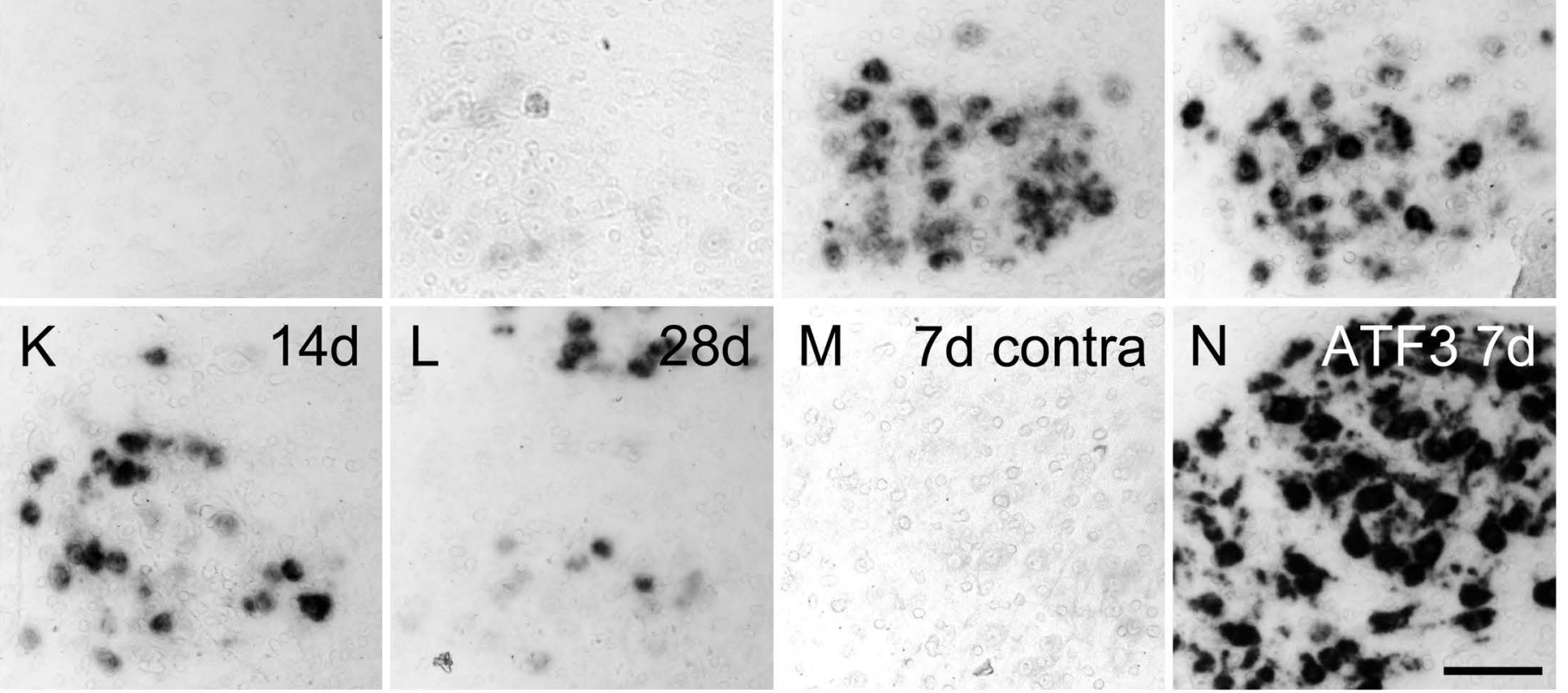

4

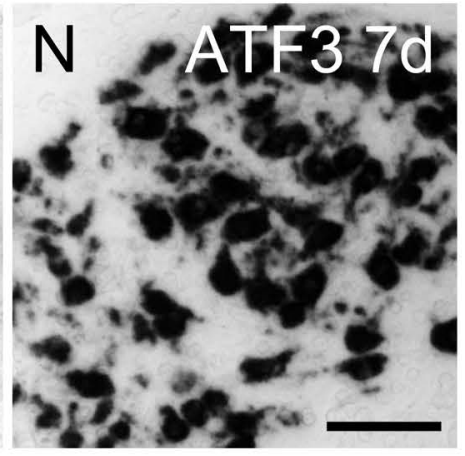




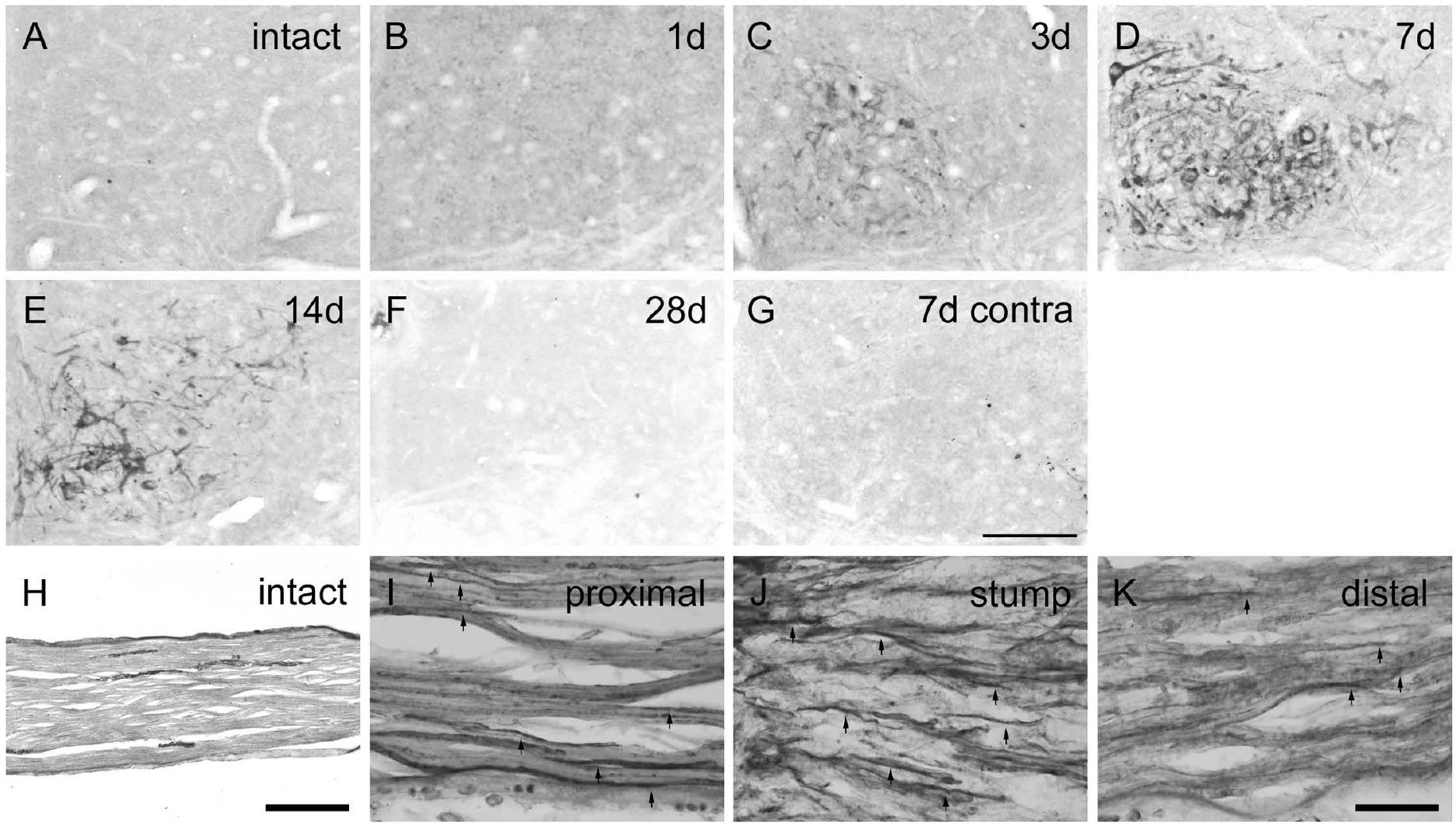




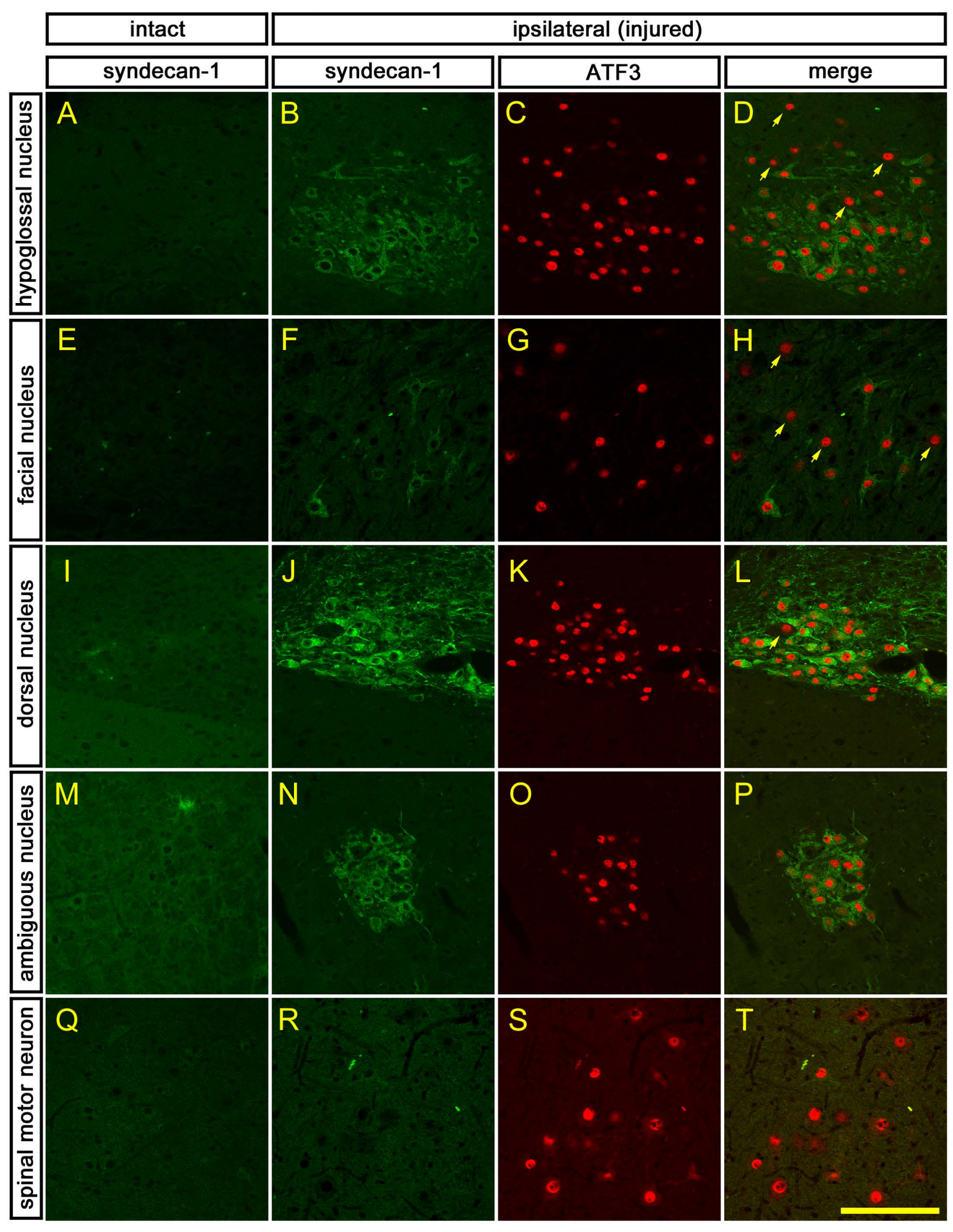




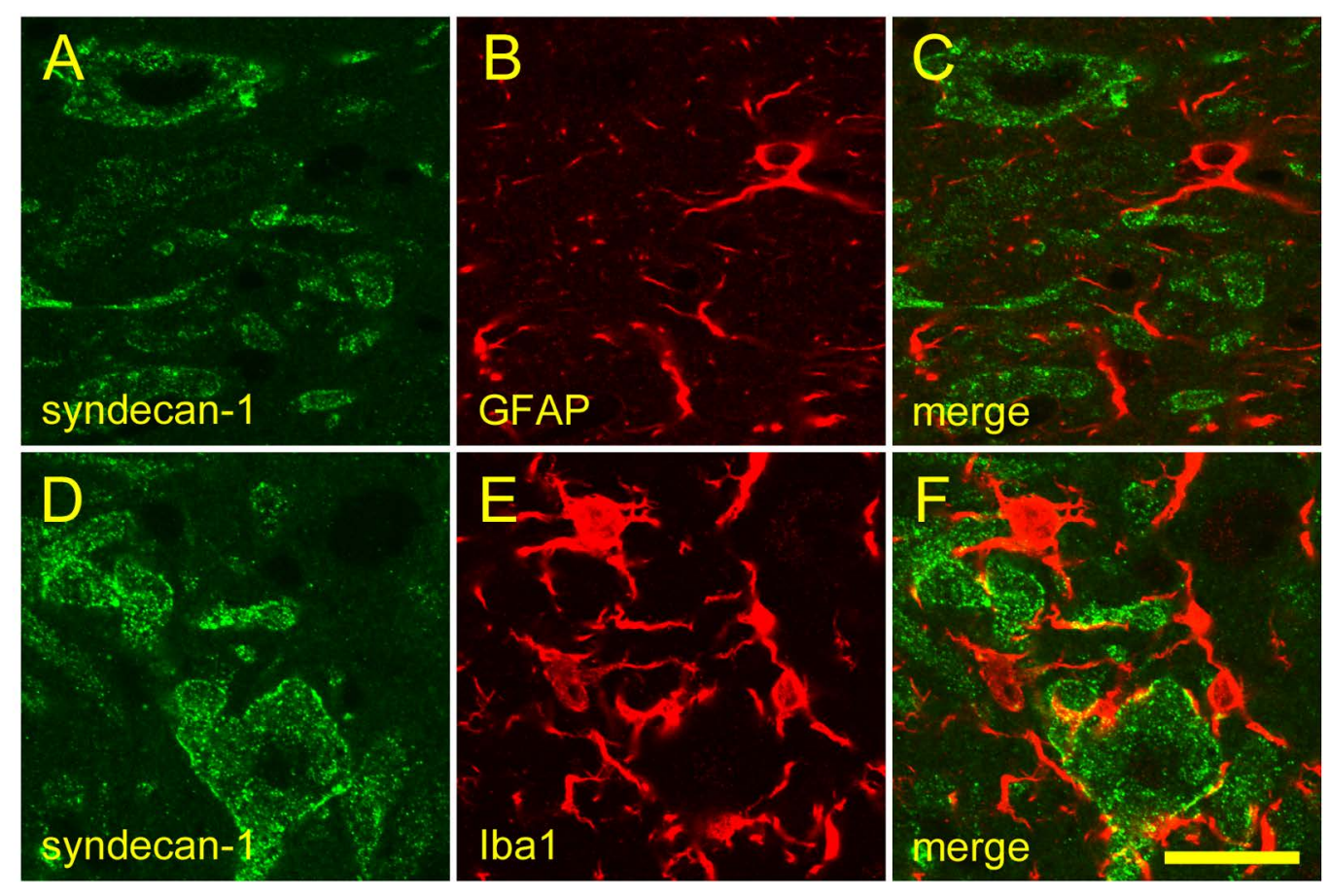




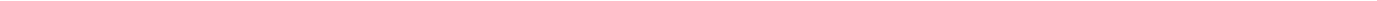




\section{contralateral}

ipsilateral

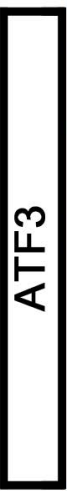

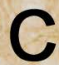

D 


\section{Supplementary Figure Legends}

Supplementary Figure 1: Immunohistochemical analysis of colocalization of syndecan-1 protein with GFAP (A-C) or Iba1 (D-F) in the hypoglossal nuclei ipsilateral to the lesion on POD7. Scale bar $=20 \mu \mathrm{m}$.

Supplementary Figure 2: Immunohistochemical analysis of colocalization of syndecan-1 protein with tubulin beta-3 chain (TUJ1) in proximal region of injured hypoglossal nerve on POD7. Scale bar $=20 \mu \mathrm{m}$.

Supplementary Figure 3: Representative images of in situ hybridization for syndecan-1 (A, B) or ATF3 (C, D) in ventral horn of spinal cord contralateral (A, C) or ipsilateral (B, D) to the lesion. Scale bar $=400 \mu \mathrm{m}$. 


\section{Highlights:}

Syndecan-1 is expressed in injured hypoglossal motor neurons.

$>$ Syndecan-1 is localized in injured neuronal cell bodies and regenerating fibers.

Spinal motor neurons scarcely express syndecan-1 after nerve injury 